\title{
Multilayer graphene grown by precipitation upon cooling of nickel on diamond
}

\author{
Jorge M. García ${ }^{\mathrm{a}, \mathrm{b}, \mathrm{c}}$, Rui He ${ }^{\mathrm{b}}$, Mason P. Jiang ${ }^{\mathrm{d}}$, Philip Kimº,d ${ }^{\mathrm{b}, \mathrm{L}}$ Loren N. Pfeiffer ${ }^{\mathrm{c}, 1}$, Aron Pinczuk ${ }^{\mathrm{b}, \mathrm{d}}$ \\ ${ }^{a}$ MBE Lab, Instituto de Microelectrónica de Madrid, IMM-CNM, CSIC, Spain \\ ${ }^{b}$ Department of Physics, Columbia University, New York, USA \\ ${ }^{c}$ Bell Labs, Murray Hill, New Jersey, USA \\ ${ }^{d}$ Department of Applied Physics and Applied Mathematics, Columbia University, New York, USA
}

\begin{abstract}
Multilayer graphene is grown by precipitation upon cooling of a thin nickel film deposited by e-beam evaporation on single crystal diamond (001) oriented substrates. Nickel acts as a strong catalyst inducing the dissolution of carbon from diamond into the metal. Carbon segregation produces multilayers of graphene on the top surface. Characterization by Raman spectroscopy reveals that these thin layers display relatively narrow Raman phonon peaks that are typically associated with graphene. Atomic force microscope measurements reveal a multigrain structure that reproduces small domains in the nickel film. The multilayer graphene is transferred onto a optical microscope glass slide for further analysis. The thickness of the layers estimated from optical transmission measurements is $12 \mathrm{~nm}$. The catalytic reaction found for nickel on diamond is not observed when glassy carbon is used as substrate. This method provides a venue for the fabrication of large area graphene films.
\end{abstract}

\section{Introduction}

The discovery of graphene and the pioneering studies of its unique physical properties by Novolesov et al. [1], sparked the enormous interest in graphene $[2,3]$. In a short time, there have been great achievements both in graphene basic science and technology that probably will soon have an impact in today's consumers society. Many of the major high tech companies have plans for the use of this material in short term plans [4]. The "graphene wave" has also re-invigorated studies in graphitic materials [5] and few graphene layer materials.

The original works employed high quality graphene obtained by mechanical cleaving of natural graphite with "scotch" tape and successive transfer to the desired substrate leading to a random deposition of graphene of small (10-100 micron) flakes with both low surface coverage and poor reproducibility. This simple method is still widely used in research and allows the observation of a variety of new physical effects in graphene [6, 7] but it is obviously not suitable for production. Major efforts in the synthesis of large-area graphene sheets have been made in recent years [8]. High-quality single- and few-layer graphene sheets have been grown by decomposition of single-crystal silicon carbide [9] and chemical vapor deposition on single-crystal transition metals such as iridium [10] and ruthenium [11, 12]. These methods require clean UHV conditions in the fabrication environment.

Carbon usually segregate in the form of graphite onto the surface of transition metals during cooling. This precipitation upon cooling process (PUC) has been known for more than

\footnotetext{
Corresponding author. Fax: +1 212854 3379. E-mail address: jorgem@imm.cnm.csic.es (J.M. García)

${ }^{1}$ Present address: Electrical Engineering Dept, Princeton University, Princeton
}

a century due to its metallurgical relevance [13, 14]. Based on PUC, an approach better suited for large-scale application has been demonstrated using an ambient-pressure chemical vapor deposition (CVD) of hydrocarbon gases on poly-crystalline transition metals. Large-area graphene sheets have been fabricated in this manner on nickel [15, 16, 17] and copper [18] thin films. With recent advances in PUC, single-layer and bilayer graphene coverage of up to $\sim 87 \%$ of the film area on nickel [19] and $~ 95 \%$ single-layer coverage on copper foils [20] has been achieved. The growth mechanism on nickel is based on gas cracking, dissolution and diffusion of atomic carbon into nickel with subsequent PUC. The growth of graphene on copper, on the other hand, is self-limiting and is a surface-catalyzed process rather than a precipitation process as it happens with nickel [21]. The graphene film can be transferred to arbitrary substrates by wet chemical etching of the underlying metal film. Another good method for the fabrication of large area graphene for transparent contact applications is the use of reduction of graphene oxide (RGO) [22].

Recently it has been demonstrated that the same PUC process can be used when the source for carbon is not an organic gas, but a beam of atomic carbon [23] in an UHV clean environment. The use of an atomic beam deposition technique [23, 24, 25], explores venues to create high quality large area graphene layers on an arbitrary substrate. In particular it would be extremely interesting to be able to produce high quality graphene layers on a thin dielectric layer placed on a conducting substrate. This Molecular Beam Epitaxy (MBE)-like approach is inspired by the great achievements in ultra high mobility $2 \mathrm{D}$ electron systems in GaAs/AlGaAs heterostructures [26].

A slightly different approach to graphene synthesis on metal surfaces has been recently reported, in which the carbon is supplied by a solid-phase method using a silicon carbide substrate 


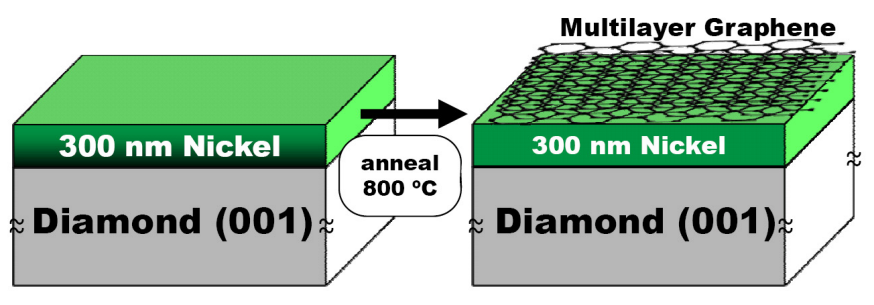

Figure 1: (Color online) Schematics of the fabrication process. After annealing a layer of $\mathrm{Ni}$ (green) on diamond at $800{ }^{\circ} \mathrm{C}$ multilayer graphene precipitates upon cooling on the surface.

as carbon source [27]. In this process a $200 \mathrm{~nm}$ nickel thin film is deposited on $\mathrm{SiC}$ via electron beam evaporation. The samples are rapidly heated in vacuum to $750{ }^{\circ} \mathrm{C}$ and immediately cooled to room temperature. During the heating process, both silicon and carbon dissolve in the nickel, forming nickel silicide saturated with atomic carbon. Upon cooling the carbon segregates to the surface of the nickel, forming single to few layers of graphene. In a similar way, it has been demonstrated that by annealing a thin CVD grown $\mathrm{SiC}$ layer [28] covered with a layer of $\mathrm{Ni}$, both silicon and carbon atoms dissolves into the metal which forms few graphene layers in a PUC process. This method has been useful in the fabrication of graphene layers and devices [28].

In brief, as it has been discussed, in a PUC synthesis of graphene the source of carbon atoms can be an organic gas which is cracked at high $\mathrm{T}[15,16,17]$, it can come from a carbon containing substrate [28] or it can be supplied by evaporation from a carbon cell [23]. The two later strategies can provide a very precise way to control the amount of carbon available for the PUC process.

In this paper we demonstrate the growth of graphene layers using diamond (001) oriented substrate as a source for carbon in a PUC process. We prove that the catalytic reaction of nickel on diamond [27, 29, 30, 31] enables the use of diamond as a carbon solid source for the fabrication of thin multilayer graphene films (see figure 1). Furthermore, we show that it is possible to combine this method with a Molecular Beam Deposition (MBD) [23] of carbon to enhance the quality of the graphene layers. The multilayer graphene film reproduces the multigrain structure of the underlying nickel film. This new method for graphene fabrication could easily be adapted for the growth of large area layers. The use of other carbon containing layers (i.e. diamond-like grown by CVD, MBD deposited layers, etc.) could make this method much more cost effective.

\section{Experimental methods}

The substrates are synthetic single crystal diamonds provided by Element $\mathrm{Six}^{T M}$ which are $3 \times 3 \times 0.5 \mathrm{~mm}^{3}$ in size and are (001)-oriented. Diamonds are employed as a solid source for carbon atoms because they are stable under high temperature treatments. The use of single crystal diamond is intended as a demonstration of the process. Currently other more cost-effective materials are being explored. Atomic Force Microscopy (AFM) measurements of the diamond show a surface roughness of $\sim 10 \mathrm{~nm}$. After the substrates are cleaned with acetone, methanol and isopropanol in an ultrasonic bath, the evaporation of metals takes place in a separate electron beam evaporation system with base pressure of $8 \cdot 10^{-6}$ mbar. The layer structure consists of $5 \mathrm{~nm}$ of titanium deposited on the diamond substrate followed by some nickel layers. Ti is used to enhance the adhesion of the Ni layer. Several samples have been processed with nickel thicknesses of 30, 80 and $300 \mathrm{~nm}$. The Raman spectra show that the D peak intensity decreases and that the $\mathrm{G}$ and $2 \mathrm{D}$ bands narrow as the thickness of the nickel layer increases up to $300 \mathrm{~nm}$ in this study. The results for the samples with a $300 \mathrm{~nm}$ thick layer of nickel are described next.

Figures 2 (a) and (b) show a representation of the temporal profiles of the annealing temperatures for samples A and B, respectively. The growth steps are carried out in an UHV system with a base pressure of $1 \cdot 10^{-10}$ mbar. During the annealing processes the pressure raises up to $3 \cdot 10^{-8}$ mbar. The sample temperature is measured by a thermocouple and by an optical pyrometer working at a wavelength of 1 micron. Further details of the experimental set up can be found elsewhere [23]. The substrates are out-gassed for 30 minutes at $800{ }^{\circ} \mathrm{C}$ (Fig. 2 (a) and (b), step $i$ ), and then they are cooled to RT. Sample A is annealed a second time to $800{ }^{\circ} \mathrm{C}$ for another $30 \mathrm{~min}$ (step iii). On sample B, a layer of carbon is deposited by means of MBD [23](Fig. 2(b), step ii) and finally it has been annealed a second time to $800{ }^{\circ} \mathrm{C}$ for $30 \mathrm{~min}$, similarly to sample A (step iii). The MBD deposited carbon thickness is $37 \AA$ and it is estimated from the carbon cell growth rate. The $C$ cell is calibrated by measuring the height of a sharp step processed with oxygen plasma etching in a non-annealed deposited thick carbon film by Atomic Force Microscope (AFM) [23]. The MBD carbon growth takes place during $300 \mathrm{~min}$ at a rate of $0.123 \AA / \mathrm{min}$. During the deposition, the substrate temperature rises to $500{ }^{\circ} \mathrm{C}$ due to the proximity to the carbon filament which is at $2100{ }^{\circ} \mathrm{C}$ (Fig. 2 (b), step ii).

The cooling rate is $-20{ }^{\circ} \mathrm{C} / \mathrm{sec}$ for the first $200{ }^{\circ} \mathrm{C}$. After the samples have been removed from the UHV chamber they are analyzed by means of Raman spectroscopy, Optical Microscope (OM) and AFM. Then, the nickel film is etched away using a selective aqueous iron chloride $\left(\mathrm{FeCl}_{3}\right)$ solution [16]. The films are then transferred onto a microscope cover glass to perform further Raman spectroscopy measurements and to estimate the thickness by means of measuring transmission absorption of a laser light.

Raman and optical absorption measurements are performed at room temperature with an argon-ion laser operating with emission at $514.5 \mathrm{~nm}$. The laser spot size is $\sim 20 \mu \mathrm{m}$ and the power is kept at $\sim 5 \mathrm{~mW}$. Spectra are recorded by a Spex-1404, 0.85-m double grating spectrometer using CCD detection. The spectral resolution is $\sim 4 \mathrm{~cm}^{-1}$. The Raman measurements focus on the well known D, G and $2 \mathrm{D}$ optical phonons. These bands are prominent features in the Raman characterization of graphite and graphene materials [32]. 

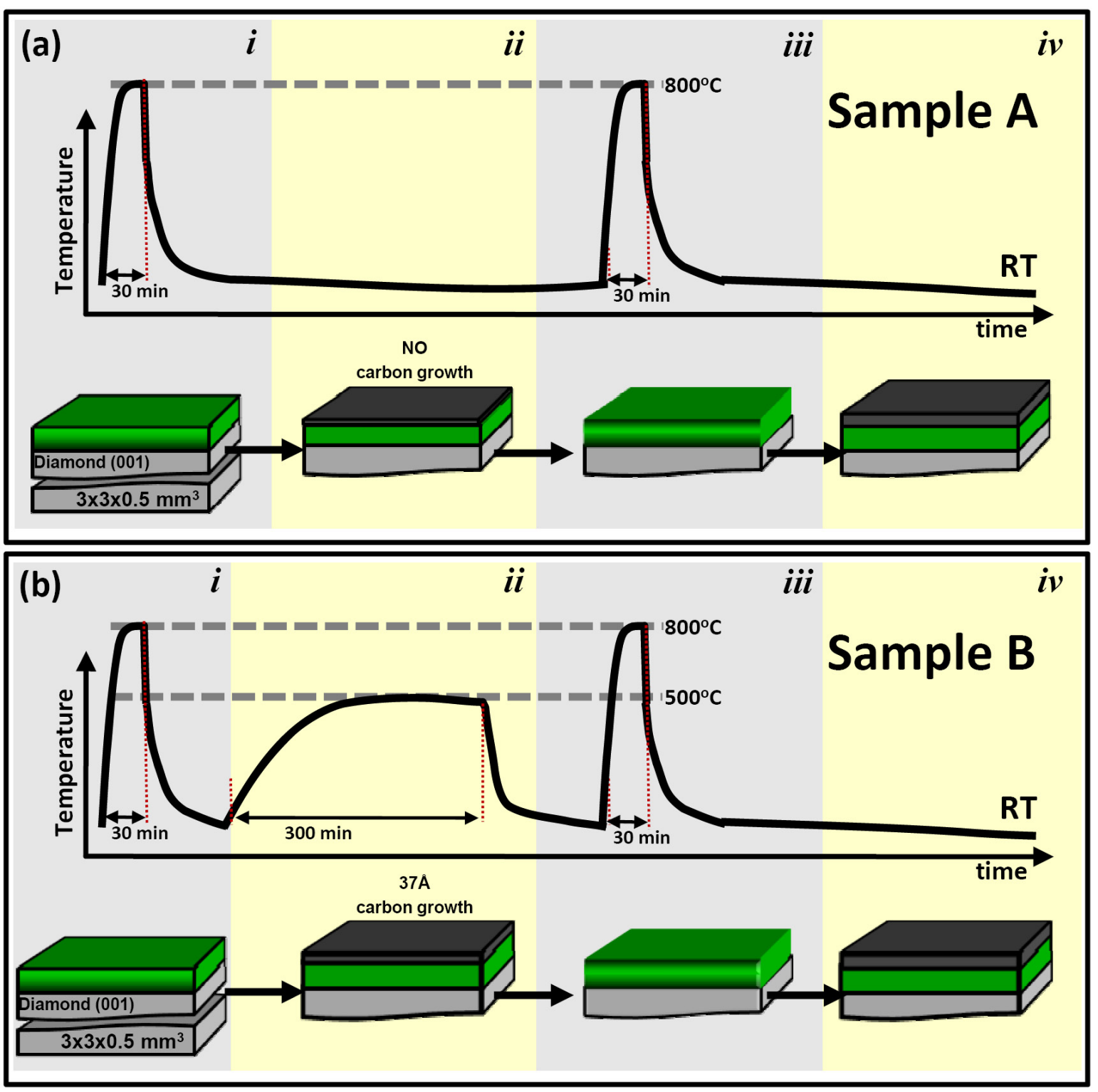

Figure 2: (Color online) Schematics of the fabrication process. After degas at $800{ }^{\circ} \mathrm{C}$ during $30 \mathrm{~min}$ for both samples ((a) and (b), step $\left.i\right)$, sample A is annealed at $800^{\circ} \mathrm{C}$ for 30 minutes (panel (a) step iii). On sample B a layer of $37 \AA$ of carbon is deposited during 300 min (panel (b), step ii). Then an annealing takes place (panel (b) step iii) at $800^{\circ} \mathrm{C}$ for $30 \mathrm{~min}$. 


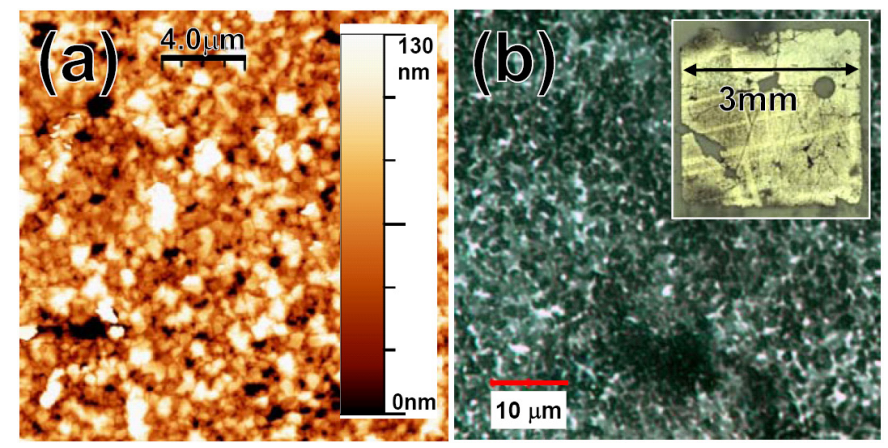

Figure 3: (Color online) (a) AFM image of the graphitic layers grown on Nickel on a diamond substrate after deposition of $37 \AA$ of atomic carbon, the lateral size of the image is $20 \times 20 \mu \mathrm{m}^{2}$ and the vertical scale is $130 \mathrm{~nm}$. (b) Optical microscope image taken with an $\times 50$ magnification on the same sample transferred onto glass with backlight illumination. The inset depicts an image of the whole sample with a 2.5 magnification objective with front light illumination.

\section{Results}

Figure 3 (a) shows an AFM image of sample B. The image is $20 \times 20 \mu \mathrm{m}^{2}$ and the color scale represents changes in height up to $130 \mathrm{~nm}$. The arithmetic average of the roughness profile, $\mathrm{R}_{a}$, is $12 \mathrm{~nm}$. The surface morphology of sample A is similar to sample B. Figure 3 (b) shows an optical microscope image taken with an $\times 50$ magnification on a sample B transferred onto glass with backlight illumination. The inset of figure 3 (b) shows the whole sample with a x2.5 magnification objective with front light illumination. Attempts to measure the thickness of the film after transferred onto glass by means of AFM or profilometry have been unsuccessful due to a very large surface roughness.

We have estimated the thickness of the transferred films by measuring its optical density with a $514.5 \mathrm{~nm}$ laser. The optical absorption is performed with a laser spot of $20 \times 20$ micron $^{2}$ in size, averaging the thickness over this area. We have performed absorption measurements in different positions of the samples and the results show a uniform (within 10\%) optical density. According to $[33,34]$ the thickness $d$ of graphene or few graphene layers can be described by

$$
d=\frac{-1}{4 \cdot \pi \cdot C_{1}} \cdot \ln \left(\frac{I}{I_{0} \cdot(1-R)}\right) ;
$$

where $C_{1}=\frac{n \kappa}{\lambda}=5.446 \mu m^{-1}, n$ is the real refractive index, $\kappa$ is the extinction coefficient, $\lambda$ is the laser wavelength, $I$ is the transmitted light intensity, $I_{0}$ is the incident light intensity, and $R$ is the fraction of reflected light which can be ignored. According to equation 1 , the thickness of the multilayer graphene film in sample A (in which all the carbon is coming from the diamond) is $d=121 \AA$. The thickness of the film in sample B (in which $37 \AA$ of carbon has been deposited by MBD) is $d=212$ A.

The Raman signal of a layer of carbon deposited by MBD on nickel on diamond with no annealing treatment shows no $\mathrm{D}, \mathrm{G}$ or $2 \mathrm{D}$ resonances (not shown, see similar spectra in samples with $\mathrm{Ni}$ on $\mathrm{SiO}_{2}$ in reference [23]). In contrast, the Raman spectra of samples A and B clearly shows those resonances and

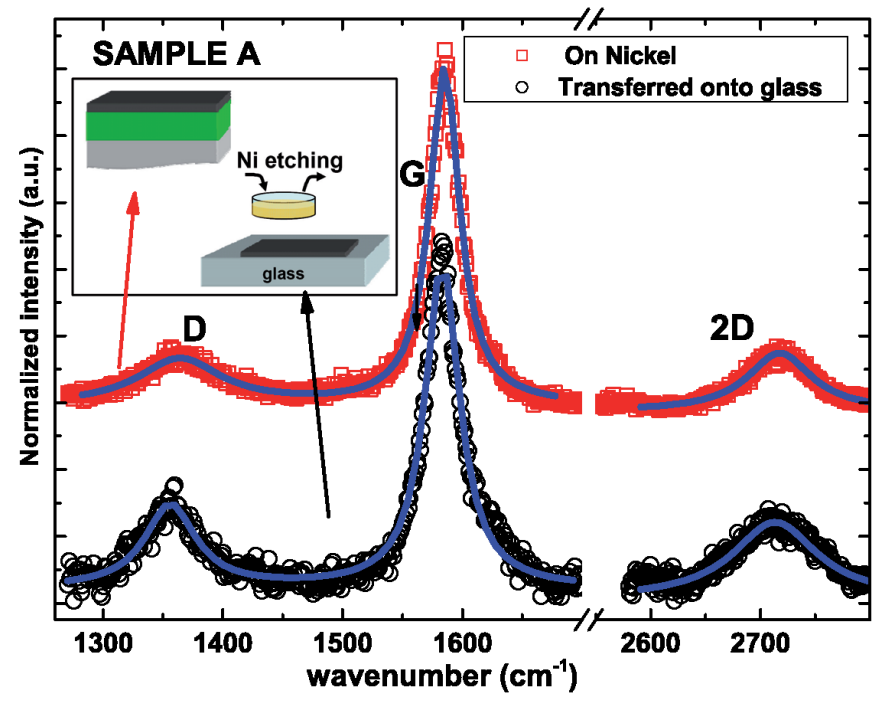

Figure 4: (Color online) Raman spectra of sample A with the multilayer graphene film on Nickel (top spectra; open red square symbols); and transferred onto a microscope cover glass (bottom spectra; open black circle symbols). Inset show schematics of the sample on $\mathrm{Ni}$ (green) and transferred onto glass after etching with $\mathrm{FeCl}_{3}$.

are shown in Fig. 4 and Fig. 5 respectively. They have been fit to Lorenztian function (color online: blue line) and normalized to the $\mathrm{G}$ resonance maximum intensity. The spectra taken on $\mathrm{Ni}$ (color online: red symbols) have been shifted up for clarity. All of them show (see table 1) Raman resonances at $\sim 1360 \mathrm{~cm}^{-1}$ (D), at $\sim 1585 \mathrm{~cm}^{-1}$ (G) and at $\sim 2715 \mathrm{~cm}^{-1}$ (2D). When a thin layer of carbon is deposited by MBD (Fig. 5, sample B), the full width at half maximum (FWHM) of the G band decreases from $30.7 \mathrm{~cm}^{-1}$ to $21.3 \mathrm{~cm}^{-1}$. The intensity of the $\mathrm{D}$ resonance in sample A increases by approximately a factor of two after the transfer.

We have observed an increase in the FWHM of the 2D peak from $75 \mathrm{~cm}^{-1}$ to $105 \mathrm{~cm}^{-1}$ when the thickness of the nickel film is decreased from $300 \mathrm{~nm}$ to $30 \mathrm{~nm}$, respectively (not shown). Currently more investigation of thickness dependence is being carried out.

We have explored other substrates and other transition metals. For example, when the annealing process employed in sample $\mathrm{A}$ is repeated using a layer of $5 / 300 \mathrm{~nm}$ of Ti/Ni on $300 \mathrm{~nm}$ of $\mathrm{SiO}_{2} / \mathrm{Si}(001)$, the Raman spectra (not showing Figs.) have no traces of the graphitic-characteristic phonon bands $(\mathrm{D}, \mathrm{G}$, and 2D).

When a glassy carbon substrate is used instead of diamond(001), no graphitic Raman phonons can be detected on the surface of the nickel. Copper has been also used as a metal buffer matrix instead of nickel, with the aim of achieving much thinner layers. Again we have not observed any Raman phonons related to graphite when samples are fabricated by employing the same annealing profiles.

\section{Discussion}

The presence of the D, G and 2D Raman resonances (Fig. 4,5 and table 1) clearly indicate the precipitation upon cool- 


\begin{tabular}{lcrrrrrrrr} 
& & \multicolumn{3}{c}{$\mathbf{D}$} & \multicolumn{2}{c}{$\mathbf{G}$} & \multicolumn{2}{c}{ 2D } \\
\cline { 3 - 10 } & $\begin{array}{c}W_{0} \\
\left(\mathrm{~cm}^{-1}\right)\end{array}$ & $\begin{array}{r}F W H M \\
\left(\mathrm{~cm}^{-1}\right)\end{array}$ & $I / I_{G}$ & $\begin{array}{c}W_{0} \\
\left(\mathrm{~cm}^{-1}\right)\end{array}$ & $\begin{array}{r}F W H M \\
\left(\mathrm{~cm}^{-1}\right)\end{array}$ & $\begin{array}{c}W_{0} \\
\left(\mathrm{~cm}^{-1}\right)\end{array}$ & $\begin{array}{r}F W H M \\
\left(\mathrm{~cm}^{-1}\right)\end{array}$ & $I / I_{G}$ \\
\hline \multirow{2}{*}{ Sample A } & On Ni & 1364.0 & 85.0 & 0.128 & 1585.2 & 30.3 & 2717,6 & 71.3 & 0.172 \\
\cline { 2 - 11 } & On Glass & 1356.1 & 47.3 & 0.251 & 1583.3 & 34.3 & 2713.5 & 92.4 & 0.234 \\
\hline \multirow{2}{*}{ Sample B } & On Ni & 1359.0 & 110.0 & 0.068 & 1585.2 & 21.3 & 2717.3 & 62.1 & 0.206 \\
\cline { 2 - 10 } & On Glass & 1357.0 & 79.9 & 0.078 & 1584.3 & 23.3 & 2714.1 & 76.8 & 0.219 \\
\hline
\end{tabular}

Table 1: Description of Raman resonances D, G and 2D on samples A and B on Ni and after transfer onto glass. $W_{0}$ is the position of the peak. FWHM is the Full Width at Half Maximum and $I / I_{G}$ is the intensity normalized to the maximum intensity of the $\mathrm{G}$ resonance.

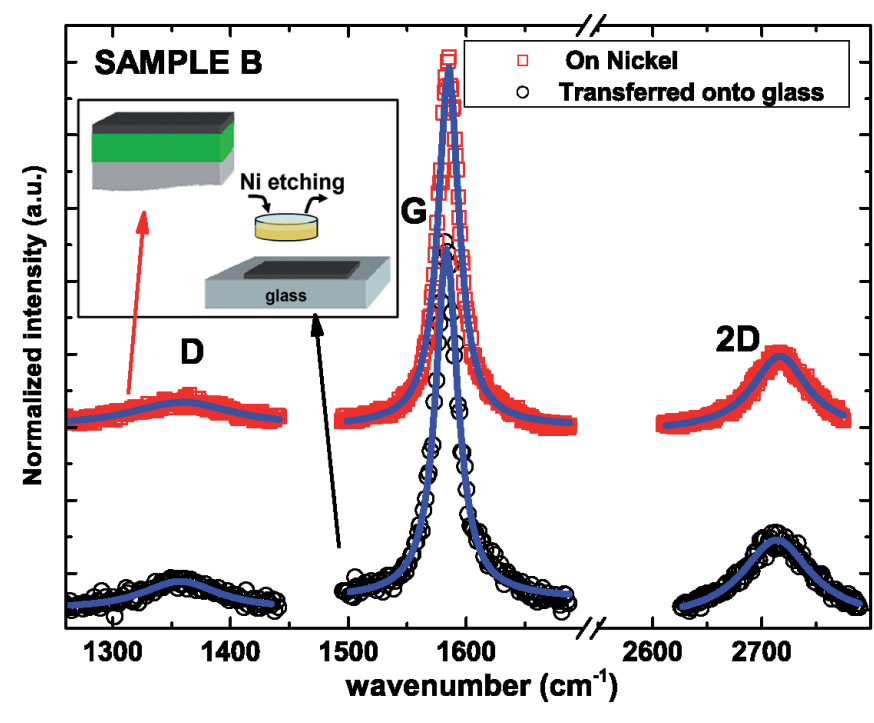

Figure 5: (Color online) Raman spectra of sample B with the multilayer graphene film on Nickel (top spectra; open red square symbols); and transferred onto a microscope cover glass (bottom spectra; open black circle symbols). Inset show schematics of the sample on $\mathrm{Ni}$ (green) and transferred onto glass after etching with $\mathrm{FeCl}_{3}$.

ing on the surface of few graphene/graphitic material. The best results are obtained for a nickel film thickness of $300 \mathrm{~nm}$. At $800^{\circ} \mathrm{C}$ carbon can diffuse through the titanium layer easily and be dissolved into the Ni. At this temperature Ti is highly soluble in $\mathrm{Ni}[35]$; therefore the nickel film will be highly exposed to the diamond. Another fact that helps the reaction of diamond with $\mathrm{Ni}$ is a diamond surface roughness which is twice as the thickness of the Ti layer.

The optical absorption measurement results show that the precipitated film in sample $\mathrm{A}$ is $\sim 36$ equivalent graphene monolayers (ML) thick $(1 \mathrm{ML} \simeq 3.35 \AA$ ). The morphology of the multilayers graphene films is imposed by the nickel multigrain structure observed in figure 3 . These multilayers of graphene reproduce the underlaying Ni grainy structure with a typical domain size $\sim 1 \mu \mathrm{m}$ (Fig. 3, a). The optical contrast variation in the image suggests also a multigrain structure with locally large variations in the optical density. The transfer process induces some damage on the sample as it can be observed in the inset of figure 3 (b). It is also apparent in figure 4, in which it can be observed that the intensity of the D peak in sample $\mathrm{A}$ is a factor two more intense after transfer. Sample B is less sensitive to the transfer, probably due to a larger thickness of the film. The large surface roughness in the film made unsuccessful thickness measurements by AFM or by optical profilometry.

The difference between the estimated thickness in sample A and in sample B is larger than the amount of deposited carbon by MBD $(\sim 37 \AA)$ and could be attributed to some extra carbon ( $\sim 54 \AA$ ) that has been dissolved into the Ni during the 5h MBD growth in which the sample has been annealed at $500{ }^{\circ} \mathrm{C}$ during the carbon deposition (see Fig. 2 (b), step ii). These results suggest that there is a strong catalatic effect of $\mathrm{Ni}$ on the dissolution of diamond, even at such low temperatures.

In comparison to other methods for the growth of large area graphene, the measurements of the D peak relative intensity in our samples are lower than those reported for Reduced Graphene Oxide (RGO) graphene grown samples [22], similar to MBE grown samples [23] but are higher than those reported for CVD grown films [16, 17]. The presence of this D band can be attributed to the high density of grain domains shown in Fig. 3. It may also be related to the presence of intrinsic defects in the grains.

The transfer process of the films onto glass induces in the $\mathrm{G}$ bandwidth a slight increase of $\sim 8 \%$ in both samples, probably due to an increase of defects or chemical residue in the film. On the other hand, when a thin layer of carbon is deposited by MBD (Fig. 5, sample B), the full width at half maximum (FWHM) of the $\mathrm{G}$ band improves from $30.7 \mathrm{~cm}^{-1}$ to 21.3 $\mathrm{cm}^{-1}$. Multilayer graphene films produced by other large area growth methods such as CVD [16] (film still on Ni) yield comparable widths on average of $26 \mathrm{~cm}^{-1}$. Alternatively, multilayer graphene grown by RGO shows a larger $\mathrm{G}$ band width of about $55 \mathrm{~cm}^{-1}$ (see supplementary information in Ref. [22]). The improvement of the quality of the samples with some MBDdeposited carbon may be related to the fact that the graphitic layers are thicker, or to the formation of pre-nucleation surface sites. It also could be related to the longer annealing time of this sample at $500^{\circ} \mathrm{C}$.

The 2D peak position $\left(2714 \mathrm{~cm}^{-1}\right)$ is markedly blue-shifted with respect to the position of the $2 \mathrm{D}$ band in a single layer graphene flake $\left(2700 \mathrm{~cm}^{-1}\right)$ but its energy is still below the one measured in bulk graphite $\left(2726 \mathrm{~cm}^{-1}\right)$. The $2 \mathrm{D}$ spectra of single graphene layer and bulk graphite have been measured in our experimental set up using the same excitation laser wavelength $(514.5 \mathrm{~nm})$. The PUC-samples 2D peak position can be interpreted by the presence of weakly coupled multilayers of graphene, or by the presence of some turbostratic order. The width of the 2D bands measured in the MBD samples (see table 1) is $62 \mathrm{~cm}^{-1}$, similar to the one measured in samples con- 
taining few graphene layers grown by CVD method [16] (64 $\mathrm{cm}^{-1}$ ), although almost twice as broad as that from a single layer graphene grown by CVD $\left(30-40 \mathrm{~cm}^{-1}\right)$ [17]. The analysis of the FWHM of the 2D Raman peak is unambiguously useful in the analysis of the number of layers of graphene [36]. According to this reference, the fullwidth of our best samples correspond to multilayer of graphene.

The final thickness of the graphitic layers will depend on the amount of dissolved carbon into nickel, on the diffusion through the nickel film and on the final segregation and nucleation on the surface. All these processes strongly depend on temperature. It should be possible to finely control the thickness of these thin graphitic layers down to a monolayer by adjusting key process parameters such as the temperature, the annealing time, cooling rate and the Ni film thickness. It is also very important to improve the nickel quality and its surface roughness. Another interesting factor to explore is the interfacial reactivity of a different diamond orientation under similar processing conditions. For example, we expect a lower catalytic reactivity of $\mathrm{Ni}$ on a (111) diamond surface, and may be another way to reduce the final graphitic layer thickness.

Our results using $\mathrm{Ni}$ on a $\mathrm{SiO}_{2} / \mathrm{Si}$ substrate instead of diamond proves that the sole source of carbon in sample $\mathrm{A}$ is the diamond(001). Also this result proves that there is no carbon contamination from the environment during the annealing process.

On the other hand, the fact that there is no dissolution of glassy carbon into nickel suggest that the catalytic effect of nickel on dissolving carbon atoms of the substrate is much larger in diamond than in glassy carbon. We can speculate that this effect is linked to the fact that this carbon allotrope is much more stable than diamond, with a much more significant amount of $\mathrm{sp}^{2}$ hybridization[37].

We have tested that copper on diamond(001) does not react in a similar way as Ni does. Therefore, this PUC growth process can not be used under similar conditions. This result can be understood based on a very low solubility of carbon on copper. Actually, it has been recently demonstrated that the growth of graphene on copper by CVD is a surface process [21].

The graphitic synthesis process proposed in this work would be much more cost-effective if diamond-like, or other carbon containing substrates, could be used. The fact that at temperatures as low as $500^{\circ} \mathrm{C}$ there is a strong reaction of diamond with nickel suggests that other substrates could be used. Experiments are currently in progress to use the carbon deposited by MBD as a source for the dissolved carbon in nickel.

\section{Conclusions}

Few graphene layers are grown by precipitation upon cooling (PUC) of a thin nickel film deposited by e-beam evaporation on a single crystal diamond (100) oriented substrates. Carbon segregation produces few graphene layers on the top surface. Characterization by Raman spectroscopy reveals that these thin graphitic films display good quality with relatively narrow Raman peaks. We also show the growth of similar samples by combination of the segregation process and a molecular beam deposited (MBD) layer of carbon. The catalytic reaction of nickel with diamond is not observed when glassy carbon is used as substrate.

\section{Acknowledgments}

We want to thank K. S. Kim for his helpful discussions and help with the transfer of the films. Also we want to thank K. West and K. Baldwin. This work is supported by ONR (N000140610138 and Graphene Muri), NSF (CHE-0117752 and CHE-0641523), NYSTAR, CSIC-PIF (200950I154), Spanish CAM (Q\&C Light (S2009ESP-1503),Numancia 2 (S2009/ENE-1477)), Spanish MICINN (NANINPHO-QD, TEC2008-06756-C03-01, Consolider QOIT (CSD2006-0019), Consolider GENESIS MEC (CSD2006-0004) and Salvador de Madariaga Grant no. PR2007-0036).

[1] Novoselov KS, Geim AK, Morozov V, Jiang D, Zhang Y, Dubonos SV, et al. Electric field effect in atomically thin carbon films. Science 2004; 306 : 666-669.

[2] Geim AK, Novoselov KS. The rise of graphene. Nature Mat. 2007; 6: 183-191.

[3] Novoselov KS, Jiang Z, Zhang Y, Morozov SV, Stormer HL, Zeitler U, et al. Room-Temperature Quantum Hall Effect in Graphene. Science 2007; 315: 1379.

[4] Sukang B, Hyeong KK, Youngbin L, Xianfang X, Jae-Sung P, Yi Z et al. 30 inch Roll-Based Production of High-Quality Graphene Films for Flexible Transparent Electrodes. arXiv:0912.5485v3

[5] Kopelevich Y, Raquet B, Goiran M, Escoffier W, da Silva R R, Medina Pantoja JC, et al. Searching for the Fractional Quantum Hall Effect in Graphite. Phys. Rev. Lett. 2009; 103: 116802-6

[6] Novoselov KS, et al. Two-dimensional gas of massless Dirac fermions in graphene. Nature 2005; 438: 197-200.

[7] Zhang Y, Tan JW, Stormer HL, Kim P. Experimental observation of the quantum Hall effect and Berry's phase in graphene. Nature 2005; 438: 201-204.

[8] Taghioskoui M, Trends in graphene research. Materials Today 2009; 12 : 34-37.

[9] Berger C, Song Z, Li X, Wu X, Brown N, Naud, et al. Electronic Confinement and Coherence in Patterned Epitaxial Graphene. Science 2006; 312: 1191-1196.

[10] N'Diaye AT, Coraux J, Plasa TN, Busse C, Michely T. Structure of epitaxial graphene on Ir (111). New J. Phys. 2008; 10: 043033-043049.

[11] Vázquez de Parga AL, Calleja F, Borca B, Passeggi MCG Jr., Hinarejos JJ, Guinea F, and Miranda R. Periodically Rippled Graphene: Growth and Spatially Resolved Electronic Structure. Phys. Rev. Lett. 2008; 100: 056807-4.

[12] Sutter PW, Fliege JI, Sutter EL. Epitaxial graphene on ruthenium. Nat. Mater. 2008; 7: 406-411.

[13] Hansen M. Constitution of Binary Alloys, McGraw-Hill Book company. $1958 ; 366$.

[14] Grant JT, and Haas TW. A study of Ru(0001) and Rh(111) surfaces using LEED and Auger electron spectroscopy. Surface Science 1970; 21: 7685.

[15] Yu Q, Lian J, Siriponglert S, Li H, Chen YP, Pei SS. Graphene segregated on Ni surfaces and transferred to insulators. Appl. Phys. Lett. 2008; 93 : 31031-31033.

[16] Kim KS, Zhao Y, Jang H, Lee SY, Kim JM, Kim KS, et al. Large-scale pattern growth of graphene films for stretchable transparent electrodes. Nature 2009; 457: 706-710.

[17] Reina A, Jia X, Ho J, Nezich D, Son H, Bulovic V, et al. Large Area, Few-Layer Graphene Films on Arbitrary Substrates by Chemical Vapor Deposition. Nano Lett. 2009; 9: 30-35.

[18] Wei D, Liu Y, Wang Y, Zhang H, Huang L, Yu G. Synthesis of N-Doped Graphene by Chemical Vapor Deposition and Its Electrical Properties. Nano Lett. 2009; 9: 1752-1758.

[19] Reina A, Thiele S, Jia X, Bhaviripudi S, Dresselhaus MS, Schaefer JA, Kong J. Growth of Large-Area Single- and Bi-Layer Graphene by Con- 
trolled Carbon Precipitation on Polycrystalline Ni Surfaces. Nano Res. 2009; 2: 509-516.

[20] Li X, Cai W, An J, Kim S, Nah J, Yang D, Piner R, et al. Large-Area Synthesis of High-Quality and Uniform Graphene Films on Copper Foils. Science 2009; 324: 1312-1314.

[21] Li X, Cai W, Colombo L, and Ruoff RS. Evolution of Graphene Growth on $\mathrm{Ni}$ and $\mathrm{Cu}$ by Carbon Isotope Labeling. Nano Lett. 2009; 9: 42684272

[22] Eda E, Fanchini G, and Chhowalla M, Nature Nanotechnol. 2008; 3: 270274.

[23] García JM, He R, Jiang MP, Yan J, Pinczuk A, Zuev YM. et al. Multilayer graphene films grown by molecular beam deposition, Solid State Comm. 2010; 150: 17-18.

[24] Al-Temimy A, Riedl C, and Starke U, Low temperature growth of epitaxial graphene on $\mathrm{SiC}$ induced by carbon evaporation. Appl. Phys. Lett. 2009; 95: 231907-2319079.

[25] Hackley J, Ali D, DiPasquale J, Demaree JD, and Richardson CJK, Graphitic carbon growth on $\mathrm{Si}(111)$ using solid source molecular beam epitaxy, Appl. Phys. Lett. 2009; 95: 133114-133116.

[26] Pfeiffer L, and West KW. The role of MBE in recent quantum Hall effect physics discoveries. Physica E 2003; 20, 57-64.

[27] Juang ZY, Wu CY, Lo CW, Chen WY, Huang C-F, Hwang et al. Synthesis of graphene on silicon carbide substrates at low temperature. Carbon 2009; 47: 2026-2031

[28] Hofrichter J, Szafranek BN, Otto M, Echtermeyer TJ, Baus M, Majerus A, et al. Synthesis of Graphene on Silicon Dioxide by a Solid Carbon Source, Nano Lett. 2010; 10: 36-42.

[29] Pitter M, Hugenschmidt MB and Behm RJ. The nickel/Diamond(100)(21)H Interface Studied with Electron Spectroscopy, Jpn. J. Appl. Phys. 1997; 36: 3635-3638.

[30] Eimori N, Mori Y, Moon J, Hatta A, Ma JS, Ito T, et al. Nickel-chemica vapour-deposited diamond interface studied by electron energy loss spectroscopy. Diamond and Rel. Mat. 1992 ; 2 : 537-541, Diamond 1992 Proceedings of the Third International Conference on the New Diamond Science and Technology, 31 March 1993.

[31] Kurimoto E, Harima H, Toda T, Sawada M, Iwami M, and Nakashima S, Raman study on the Ni/SiC interface reaction; J. Appl. Phys. 2002; 91: 10215-10217.

[32] Ferrari AC, Meyer JC, Scardaci V, Casiraghi C, Lazzeri M, Mauri F, et al., Phys. Rev. Lett. 2006; 97: 187401-4.

[33] Bruna $\mathrm{M}$ and Borini S. Optical constants of graphene layers in the visible range. Appl. Phys. Lett. 2009; 94: 031901-031903.

[34] Johnson LG, and Dresselhaus G, Phys. Rev. B 1973; 7: 2275-2285.

[35] Brantley WA, Iijima M, and Grentzer TH. Thermochimica Acta 2002; 392-393:329-37.

[36] Lee DS, Riedl, Krauss, von Klitzing K, Starke U, and Smet JH. Nano Lett. 2008; 8(12): 4320-4325.

[37] Harris PJF. Fullerene-related structure of commercial glassy carbons. Phil. Mag. 2004; 84: 3159-3167. 